\title{
Differential expression of inducible nitric oxide synthase in keratocystic odontogenic tumors prior and subsequent to decompression
}

\author{
WEI XU ${ }^{1,2 *}$, XIAOMENG SONG ${ }^{1,3^{*}}$, XIAOMIN ZHANG ${ }^{1,4}$, ZHAO WANG $^{1,3}$, \\ XU DING ${ }^{1,3}$, YE YUAN ${ }^{1,3}$, YUNONG WU ${ }^{1,3}$ and HEMING WU ${ }^{1,3}$ \\ ${ }^{1}$ Jiangsu Key Laboratory of Oral Diseases, Nanjing Medical University, Nanjing, Jiangsu 210029; ${ }^{2}$ Department of Stomatology, \\ First People's Hospital of Lianyungang, Lianyungang, Jiangsu 222000; Departments of ${ }^{3}$ Oral and Maxillofacial Surgery and \\ ${ }^{4}$ Pediatric Dentistry, Affiliated Hospital of Stomatology, Nanjing Medical University, Nanjing, Jiangsu 210029, P.R. China
}

Received June 3, 2015; Accepted October 29, 2015

DOI: $10.3892 /$ mco.2016.751

\begin{abstract}
The aim of the present study was to investigate the expression of inducible nitric oxide synthase (iNOS) in keratocystic odontogenic tumors (KCOTs) prior and subsequent to decompression and to explore the association between iNOS expression and changes in clinical features. Sixteen pairs of specimens obtained at the time of decompression and subsequent curettages were collected and immunohistochemically examined using an antibody against iNOS. The intensity of iNOS staining was evaluated semi-quantitatively for statistical analysis. Prior to decompression, $87.5 \%$ of KCOT samples showed no immunohistochemical reactivity for iNOS. Only $12.5 \%$ of samples exhibited slight staining for iNOS in the cytoplasm of cells in the epithelial layer. Subsequent to decompression, all the samples exhibited moderate to intense staining for iNOS in the cytoplasm and membrane of cells in the epithelial and fibrous layers. This increased expression of iNOS following decompression was statistically significant $(\mathrm{P}<0.01)$. The results demonstrated distinct expression of iNOS in KCOT samples prior and subsequent to decompression, indicating that iNOS may have a role in mediating changes in clinical features.
\end{abstract}

Correspondence to: Professor Heming Wu or Professor Yunong Wu, Jiangsu Key Laboratory of Oral Diseases, Nanjing Medical University, 140 Hanzhong Road, Nanjing, Jiangsu 210029, P.R. China

E-mail:whmz2002@aliyun.com

E-mail: yunongwu@aliyun.com

${ }^{*}$ Contributed equally

Key words: decompression, keratocystic odontogenic tumor, inducible nitric oxide synthase

\section{Introduction}

Keratocystic odontogenic tumors (KCOTs) are one of the most common odontogenic tumors of the jaw. Previously known as odontogenic keratocysts (OKCs), these tumors were reclassified by the World Health Organization in 2005 (1). KCOTs generally appear as unilocular or multilocular radiolucencies with a smooth border or honeycomb appearance, and they are characterized by an aggressive behavior and a high rate of recurrence. Currently, decompression or marsupialization, combined with stage-two curettage or enucleation, represent the commonly accepted treatment approach for KCOTs; the advantage of this therapeutic approach is its minimally invasive nature, which allows for preservation of jaw function and appearance. Following decompression, the size of the lesion may decrease (2). Furthermore, a previous study (3) showed that the histological features of KCOTs markedly changed following decompression, as evidenced by the thickening of the epithelial layer and enhanced inflammation in the fibrous layer. However, the mechanisms responsible for these changes in clinical features remain to be elucidated.

Inducible nitric oxide synthase (iNOS) is a cytosolic enzyme that has been closely associated with the pathophysiological process of inflammatory diseases, such as periodontal disease (4) and periapical inflammatory lesion (5). NO is produced mainly by iNOS. Watanuki et al (6) showed that NO generated by iNOS in osteoblasts has a critical role in regulating bone turnover and elevated osteogenic activity, and Chen et al (7) reported iNOS expression in KCOT. However, to the best of our knowledge, no studies have investigated how iNOS expression changes in response to decompression treatment. In the present study, immunohistochemistry was used to detect the expression of iNOS in KCOT samples obtained prior and subsequent to decompression and to assess the possible roles of iNOS in mediating changes in clinical features.

\section{Materials and methods}

Patients and tissue samples. A total of 16 histologically verified KCOT specimens collected between 2004 and 2009 were 
Table I. Clinical information and the expression intensity of iNOS.

\begin{tabular}{llccllll}
\hline & & & & & & \multicolumn{2}{c}{ iNOS expression, $\mathrm{n}$} \\
\cline { 5 - 8 } Patient & Gender & Age, years & Duration, months & Location & Radiographic features & BC & AC \\
\hline 1 & Male & 22 & 27 & Mandible; Mol-Ram & Solitary; unilocular & 0 & 9 \\
2 & Male & 15 & 10 & Mandible; Ang-Ram & Multiple; unilocular & 0 & 4 \\
3 & Male & 20 & 21 & Mandible; Ang-Ram & Solitary; unilocular & 0 & 9 \\
4 & Male & 42 & 17 & Mandible; Mol-Ram & Multiple; unilocular & 0 & 6 \\
5 & Female & 20 & 16 & Mandible; Ang-Ram & Solitary; unilocular & 0 & 4 \\
6 & Female & 13 & 12 & Mandible; Mol-Ram & Solitary; unilocular & 0 & 4 \\
7 & Female & 38 & 3 & Mandible; Ang-Ram & Solitary; multilocular & 0 & 6 \\
8 & Female & 34 & 16 & Mandible; Mol-Ram & Solitary; unilocular & 2 & 9 \\
9 & Female & 49 & 18 & Mandible; Ang-Ram & Solitary; unilocular & 0 & 4 \\
10 & Male & 55 & 15 & Maxilla; Ant & Multiple; unilocular & 0 & 4 \\
11 & Female & 25 & 15 & Mandible; Mol-Ram & Solitary; unilocular & 0 & 4 \\
12 & Female & 33 & 9 & Mandible; Ang-Ram & Solitary; unilocular & 0 & 6 \\
13 & Male & 35 & 23 & Mandible; Ang-Ram & Solitary; multilocular & 1 & 4 \\
14 & Female & 24 & 31 & Mandible; Ang-Ram & Solitary; multilocular & 0 & 6 \\
15 & Male & 29 & 23 & Maxilla; Ant & Solitary; unilocular & 0 & 6 \\
16 & Female & 28 & 27 & Maxilla; Ant & Multiple; unilocular & 0 & 6 \\
\hline
\end{tabular}

0, no staining; 1-3, slight staining; 4-6, moderate staining; $>6$, intense staining. iNOS, inducible nitric oxide synthase; BC, before decompression; AC, after decompression; Ant, anterior region; Mol, morlar region; Ang, angular region; Ram, mandiblular ramus.
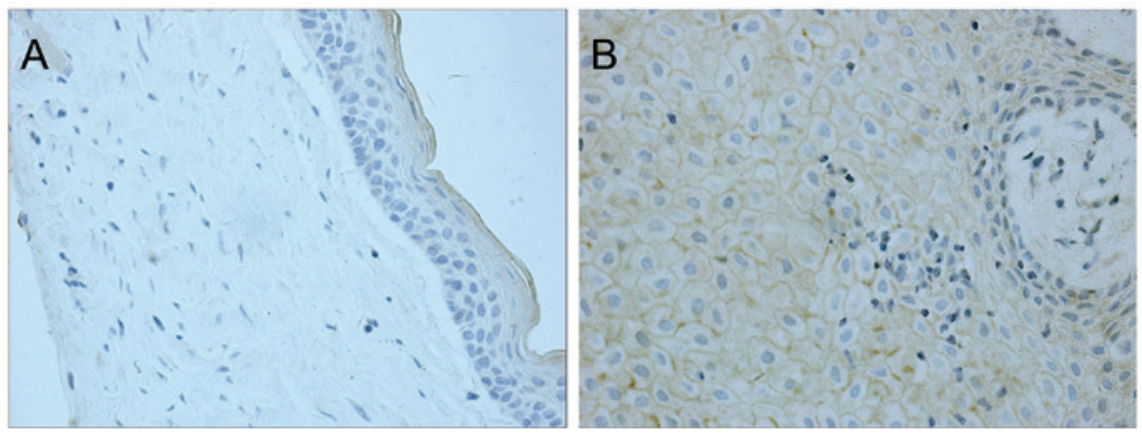

Figure 1. Immunohistochemical reactivity for inducible nitric oxide synthase in keratocystic odontogenic tumor samples obtained (A) prior and (B) subsequent to decompression in the same subject (magnification, $\mathrm{x} 400$ ).

obtained from the Stomatology Hospital of Jiangsu Province (Jiangsu, China) and from the Shanghai Ninth People's Hospital (Shanghai, China). Recurrent cases or those associated with nevoid basal cell carcinoma syndrome were excluded from the study. All the patients were treated by decompression followed by enucleation. The clinical information of the patients is shown in Table I. Postoperative follow up consisted of clinical and radiographic examinations. The mean duration before stage II surgery was 19.5 months (range, 6.5-44.0 months).

Immunohistochemistry. Paraffin specimens obtained at the time of decompression and enucleation were collected from the Department of Oral Pathology at the Stomatology Hospital of Jiangsu Province and the Shanghai Ninth People's Hospital. Each of the 16 pairs of paraffin-embedded samples was sectioned serially into two $4-\mu \mathrm{m}$ slices; one slice was prepared for immunohistochemical analysis, while the other was used as a negative control by substituting phosphate-buffered saline (PBS) for the specific antibody. Briefly, the deparaffinized sections were immersed in absolute methanol containing $3 \%$ hydrogen peroxide for $15 \mathrm{~min}$ at room temperature to block endogenous peroxidase activity. Following washing with PBS, the sections were immersed in $0.01 \mathrm{M}$ citrate buffer ( $\mathrm{pH}$ 6.0), and heated in a microwave oven at $95^{\circ} \mathrm{C}$ for $5 \mathrm{~min}$. Subsequently, diluted (1:50) mouse monoclonal anti-iNOS antibody (cat. no. ab195661; mouse anti-human; Abcam, Cambridge, UK) was applied to the sections at $4^{\circ} \mathrm{C}$ overnight. The sections were subsequently incubated with rabbit-anti-mouse secondary antibody (1:5,000; Abcam) for $30 \mathrm{~min}$ at room temperature. The sections were immersed for $8 \mathrm{~min}$ in $0.03 \%$ 3,3-diaminobenzidine tetrahydrochloride in $0.05 \mathrm{M}$ Tris- $\mathrm{HCl}$ buffer (pH 8.5) containing $0.01 \%$ hydrogen peroxide and counterstained with hematoxylin. 
Immunohistochemical evaluation. The immunohistochemical staining pattern of iNOS in KCOT samples appeared as brown granules on the cell membrane and in the cytoplasm. Immunohistochemical reactivity for iNOS was defined as the proportion score multiplied by the intensity score. The proportion score was defined as $0,<5 \% ; 1,6-25 \% ; 2,26-75 \%$; or $3,>76 \%$ positive cells. The intensity score was defined as 0 , negative; 1 , weak; 2 , moderate; or 3 , strong. The total score ranged from 0 to 9 . The immunoreactivity scores were used to classify the samples into one of the following three groups based on the final score: Negative immunoreactivity, defined as a total score of 0 ; low expression, defined as a total score of 1-3; moderate expression, defined as a total score of 4-6; and high expression, defined as a total score of $>6$.

Statistical analyses. Statistical analyses were performed using the paired t-test to evaluate differences in iNOS immunoreactivity in KCOTs at the time of enucleation compared to the time of decompression. $\mathrm{P}<0.05$ was considered to indicate a statistically significant difference.

\section{Results}

iNOS staining. Prior to decompression, only slight iNOS staining, which was restricted to the cytoplasm, was observed in $2(12.5 \%)$ of the 16 cases; no immunostaining was observed in the other samples (Fig. 1A, Table I). Following decompression, all the samples exhibited moderate to strong staining for iNOS in the cytoplasm and on the cell membrane of cells in the epithelial layer (Fig. 1B, Table I). In addition, the fibrous layer also showed positive iNOS expression following decompression. This increase in iNOS expression following decompression was statistically significant $(\mathrm{P}<0.01)$.

\section{Discussion}

KCOTs are one of the most frequent odontogenic tumors and they receive significant attention due to their aggressive biological behavior and tendency for recurrence (8). Decompression is commonly employed as a conservative treatment for KCOT. Following decompression, the size of the tumor is significantly decreased. In particular, Nakamura et al (9) reported that $96 \%$ of the cases in their study showed a cystic reduction $>50 \%$. Furthermore, it was reported that the typical features of KCOTs are significantly altered following decompression treatment (9). In our previous study (3) we observed that subsequent to decompression, the typical presentation of KCOT was altered to one marked by hyperplastic epithelium, thickened fibrous lamina and increased inflammatory infiltration. Numerous studies have shown that biomarkers typically expressed at high levels in KCOTs, such as interleukin (IL)-1 $\alpha$, collagenase, Ki-67, B-cell lymphoma-2 and keratinocyte growth factors, are notably decreased following decompression (10-12) indicating the attenuation of cell proliferation, survival and local invasion. Ninomiya et al (13) further showed that the expression of IL- $1 \alpha$ mRNA and protein in epithelial cells of KCOTs was significantly decreased following marsupialization and that the Ki-67 labeling index decreased proportionally with the expression of IL-1 $\alpha$. These results suggest that marsupialization may reduce the size of KCOTs by inhibiting IL-1 $\alpha$ expression and epithelial cell proliferation.

iNOS is a cytosolic enzyme induced by cytokines and bacterial lipopolysaccharide during inflammation (14). Furthermore, NO is generated primarily by iNOS and has been shown to regulate inflammation (15). Numerous studies have demonstrated that the activation of iNOS is associated with the pathophysiological characteristics of inflammatory diseases $(4,5)$. NO may also participate in the regulation of bone reconstruction. For instance, Ralston et al (16) showed that higher concentrations of NO inhibited bone resorption, whereas lower concentrations of NO stimulated bone resorption. However, the mechanisms underlying the expression of iNOS in inflammation and the regulation of bone metabolism following decompression remain to be elucidated.

To date, few studies have investigated the differential expression of iNOS in KCOTs prior and subsequent to decompression. In the present study, $87.5 \%$ of KCOT samples showed no immunohistochemical reactivity for iNOS prior to decompression, and only $12.5 \%$ of samples showed slight staining in the cytoplasm of cells in the epithelial layer. Similarly, Chen et al (7) observed iNOS expression in $10 \%$ of OKC samples. Following decompression, all the samples in the present study exhibited moderate to intense staining for iNOS in the cytoplasm and on the cell membrane of cells in the epithelial and fibrous layers. Thus, the significantly distinct expression of iNOS prior and subsequent to treatment suggests an important role for this enzyme in the progression of inflammation and the effects of KCOT decompression. However, more samples are required to further validate these results in future studies.

\section{References}

1. Barnes L, Eveson JW, Reichart P, et al: World Health Organization Classification of Tumors. Pathology and Genetics of Head and Neck Tumors. IARC Press, Lyon, 2005.

2. Shudou H, Sasaki M, Yamashiro T, Tsunomachi S, Takenoshita Y, Kubota Y, Ninomiya T, Kawazu T and Mori Y: Marsupialisation for keratocystic odontogenic tumours in the mandible: Longitudinal image analysis of tumour size using 3D visualised CT scans. Int J Oral Maxillofac Surg 41: 290-296, 2012.

3. Morais de Melo W, Pereira-Santos D, Sonoda CK and Hochuli-Vieira E: Decompression for management of keratocystic odontogenic tumor in the mandible. J Craniofac Surg 23: e639-e640, 2012.

4. Güllü C,Ozmeric N, Tokman B, Elgün S and Balos K: Effectiveness of scaling and root planing versus modified Widman flap on nitric oxide synthase and arginase activity in patients with chronic periodontitis. J Periodontal Res 40: 168-175, 2005.

5. Suzuki T, Kumamoto H, Ooya K and Motegi K: Expression of inducible nitric oxide synthase and heat shock proteins in periapical inflammatory lesions. J Oral Pathol Med 31: 488-493, 2002.

6. Watanuki M,Sakai A, Sakata T, Tsurukami H, Miwa M,Uchida Y, Watanabe K, Ikeda K and Nakamura T: Role of inducible nitric oxide synthase in skeletal adaptation to acute increases in mechanical loading. J Bone Miner Res 17: 1015-1025, 2002.

7. Chen WL, Ouyang KX, Li HG, Huang ZQ, Li JS and Wang JG: Expression of inducible nitric oxide synthase and vascular endothelial growth factor in ameloblastoma. J Craniofac Surg 20: $171-175,2009$.

8. Bhargava D, Deshpande A and Pogrel MA: Keratocystic odontogenic tumour (KCOT)-a cyst to a tumour. Oral Maxillofac Surg 16: 163-170, 2012.

9. Nakamura N, Mitsuyasu T, Mitsuyasu Y, Taketomi T, Higuchi Y and Ohishi M: Marsupialization for odontogenic keratocysts: Long-term follow-up analysis of the effects and changes in growth characteristics. Oral Surg Oral Med Oral Pathol Oral Radiol Endod 94: 543-553, 2002. 
10. Suyama Y, Kubota Y, Yamashiro T, Ninomiya T, Koji T and Shirasuna K: Expression of keratinocyte growth factor and its receptor in odontogenic keratocysts. J Oral Pathol Med 38: 476-480, 2009.

11. Pogrel MA and Jordan RC: Marsupialization as a definitive treatment for the odontogenic keratocyst. J Oral Maxillofac Surg 62: 651-655, 2004.

12. August M, Faquin WC, Troulis MJ and Kaban LB: Dedifferentiation of odontogenic keratocyst epithelium after cyst decompression. J Oral Maxillofac Surg 61: 678-683, 2003.

13. Ninomiya T, Kubota Y, Koji T and Shirasuna K: Marsupialization inhibits interleukin-1alpha expression and epithelial cell proliferation in odontogenic keratocysts. J Oral Pathol Med 31: 526-533, 2002.
14. Bredt DS, Hwang PM, Glatt CE, Lowenstein C, Reed RR and Snyder SH: Cloned and expressed nitric oxide synthase structurally resembles cytochrome P-450 reductase. Nature 351: 714-718, 1991.

15. Kendall HK, Marshall RI and Bartold PM: Nitric oxide and tissue destruction. Oral Dis 7: 2-10, 2001.

16. Ralston SH, Ho LP, Helfrich MH, Grabowski PS, Johnston PW and Benjamin N: Nitric oxide: A cytokine-induced regulator of bone resorption. J Bone Miner Res 10: 1040-1049, 1995. 